\title{
Emotional, Behavioral, and Cognitive Correlates of Attention Deficit and Hyperactive Disorder (ADHD) Screening and Diagnosis History: Sex/Gender Differences
}

\author{
Shervin Assari ${ }^{1,2 *}$ \\ 'Department of Urban Public Health, Charles R. Drew University of Medicine and Science, Los Angeles, CA, USA \\ ${ }^{2}$ Department of Family Medicine, Charles R. Drew University of Medicine and Science, Los Angeles, CA, USA
}

\section{Article Info}

\section{Article Notes}

Received: November 20, 2020

Accepted: February 01, 2021

\section{*Correspondence:}

Dr. Shervin Assari, Department of Urban Public Health, Charles R. Drew University of Medicine and Science, Los Angeles, CA USA; Telephone No.: +(734)-232-0445; Fax No: +734-615-873; Email: assari@umich.edu.

(C) 2021 Assari S. This article is distributed under the terms of the Creative Commons Attribution 4.0 International License

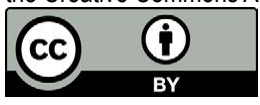

\section{Keywords:}

Attention Deficit and Hyperactive Disorder (ADHD)

Internalizing Problem Behaviors

Externalizing Behavioral Problems

Children

Sex

Cognition

\section{ABSTRACT}

Background: While clinical studies have documented sex differences in emotional, behavioral, and cognitive function of children with Attention Deficit and Hyperactive Disorder (ADHD), it is unknown if these sex differences are due to differences in referral and diagnosis or if they can be also seen when we screen a community sample for ADHD. If these sex differences exist in populations with a diagnosis history but cannot be seen in screening, then they are unfair, preventable, and due to gender (social processes in referral and diagnosis) rather than sex.

Aim: Using the data from a community sample of 9-10-year-old healthy developing children, we explored sex differences in the associations between cognitive, emotional, behavioral, and health status with positive screening vs. history of diagnosed ADHD.

Methods: The Adolescent Brain Cognitive Development (ABCD) study included a national sample of 10,171 American children between ages 9 and 10 years old. This sample included 1,488 children with a history of psychiatric diagnosis and 8,683 children without a diagnosis. The two independent variables were screening and history of ADHD. The following variables were outcomes: symptom severity, cognitive function, body mass index (BMI), internalizing, externalizing, and total behavioral disorders. Sex was the moderator, and age, race, ethnicity, education, household income, and family structure were covariates. Mixed-effects regression models were used to adjust for the nested nature of the data.

Results: Positive screening for ADHD and a history of diagnosis were both associated with worse cognitive function, higher internalizing, externalizing, total problem behaviors, higher inattention (ADHD symptoms), and lower BMI. Sex altered the association between history of diagnosis but not positive screening for ADHD with externalizing, and total behavioral problems as well as cognitive function. Sex did not affect the associations between positive screening for ADHD or a history of diagnosis with BMI or ADHD symptoms. Both history of diagnosis and positive screening for ADHD were associated with higher internalizing for boys than girls.

Conclusion: History of diagnosis, but not positive screening for ADHD, is differently associated with behavioral and cognitive performance of males and females. As sex differences are seen in correlates of history of diagnosis but not positive screening, some of the observed sex differences are due to differential referral and diagnosis rather than differential presentation of ADHD in the community. This finding suggests that some of the so-called "sex differences" that are believed to be due to biology and heritable may be "gender differences"and modifiable. This is important because while gender differences are preventable and modifiable, sex differences are not.

\section{Introduction}

Attention Deficit and Hyperactive Disorder (ADHD) is one of the 
most common psychiatric disorders during childhood ${ }^{1}$ which may last into adulthood ${ }^{2}$. Although the etiology of ADHD is not completely known, multiple studies suggest strong genetic heritability to ADHD ${ }^{3,4}$. Research has also established the role of maternal factors such as lead exposure $^{3}$, alcohol ${ }^{4,5}$, tobacco ${ }^{4}$, and history of preterm delivery ${ }^{4}$.

One of the most prominent issues in children with ADHD is its association with a wide range of behavioral, cognitive, and emotional problems ${ }^{2,8,9}$. ADHD accompanies difficulties with paying attention, inhibition, impulse control, and both internalizing and externalizing problems ${ }^{2,8,9}$. Some of this burden is core elements of ADHD and some are comorbid conditions to ADHD. For example, While poor executive function ${ }^{6}$ and impulsivity ${ }^{7,8}$ are believed to be core to ADHD, high internalizing ${ }^{9-11}$, externalizing ${ }^{11-14}$, and total behavioral problems ${ }^{15,16}$ are commonly seen as comorbidities with ADHD.

Boys and girls with ADHD show different behavioral, emotional, and cognitive profiles, causing differential burden and the manifestation of ADHD for boys and girls ${ }^{17}$. Meta-analyses have asked to what degree these differences are biological (sex differences) or due to society (gender differences $)^{17}$. Sex differences are due to biological factors that are predominantly shaped by brain differences, hormones, or sex chromosomes. On the other hand, gender differences are shaped by social forces that are preventable. Still, it is not clear whether differences in behavioral, emotional, and cognitive correlates of $\mathrm{ADHD}^{18-21}$ are due to biology or society ${ }^{22-27}$. While boys show a higher prevalence of ADHD than girls, at least in theory, some of the differential correlates of $\mathrm{ADHD}^{8,24,28-30}$ between the sexes may be due to differential referral or diagnosis. If differential referral contributes to this phenomenon, we expect larger malefemale differences in depression, impulsivity, cognitive function, and problem behaviors ${ }^{24-26,31,32}$ in children with a history of diagnosed ADHD, compared to children who screen positive for ADHD in community-based studies.

\section{Aims}

In this investigation, we analyzed a national sample of 9-10-year-old American children for the association between history of diagnosis and positive screening for ADHD with a wide range of emotional, behavioral, and cognitive outcome measures. While ADHD is expected to be correlated with internalizing behavioral problems, externalizing behavioral problems, total behavioral problems, and domains of cognitive function, we expected stronger differences between male than female children for history of diagnosis rather than positive screening for ADHD. If our hypothesis is confirmed, we would suggest that at least some of the male-female differences in correlates of ADHD are due to differential referral and diagnosis (gender differences due to society) rather than non-preventable sex differences that are inherent to the biology of ADHD.

\section{Methods}

\section{Design and Setting}

This cross-sectional study was a secondary analysis of existing data, which was borrowed from the Adolescent Brain Cognitive Development (ABCD) study ${ }^{33-37}$. The $\mathrm{ABCD}$ is a national children's brain development study with large diversity based on race, ethnicity, sex, and socioeconomic status SES ${ }^{33,38}$. The ABCD children were drawn from schools across multiple cities nested to various states in the US. The recruitment catchment area of the ABCD study sites composes 21 participating sites, encompassing over $20 \%$ of the entire United States population of 9-10-year-old children. Although the ABCD sample is national, it is not random or representative. Although the selection was not random, the final ABCD sample is a close approximation of the US children across all demographic and sociodemographic factors due to carefully designed and implemented procedures. Thus, the results are reliable regarding race/ethnicity, age, sex, SES, and urbanicity.

This study included 10,171, 9-10-year-old American children who had valid data on ADHD, sex, BMI, CBCL scores, cognitive performance, age, race, ethnicity, parental education, household income, and family structure. Participants and their families could identify as White, Black, Asian, other/mixed race, Hispanic/Latino, or nonHispanic/Latino. No participants were excluded for any reason other than missing data.

\section{Study Measures}

\section{Independent Variables}

Current ADHD Diagnosis. Parents were interviewed using the Kiddie Schedule for Affective Disorders and Schizophrenia (K-SADS) inventory to determine whether the child meets the criteria for current ADHD. The K-SADS is an interview tool that is commonly used to evaluate a wide range of psychiatric disorders in children. Application of K-SADS to parents, we had a dichotomous variable that reflected whether the child currently had ADHD.

History of $A D H D$ diagnosis. Parents were asked, "Has your child been diagnosed with ADHD, Depression, Bipolar Disorder, Anxiety, Phobias". Responses were binary and could be either yes or no.

\section{Outcomes}

The Child Behavior Checklist (CBCL). The CBCL scale ${ }^{39-42}$ was used to measure inattention as well as internalizing, externalizing, and total behavioral disorders of the children. 
In this study, the CBCL was reported by the parents ${ }^{43-49}$. The CBCL is developed by the Achenbach System of Empirically Based Assessment (ASEBA) and provides continuous scores that reflect internalizing, externalizing, and total behavioral problems. The CBCL is a useful screening tool widely used across settings for detecting children with internalizing and externalizing behavioral issues. Higher scores indicate more internalizing, externalizing, and total behavioral problems. ${ }^{39-42}$

BMI. Child BMI was measured up to two times. BMI was calculated based on measured height and weight.

Cognitive Function. Using the NIH toolbox, we had three measurement tools for assessing the neurocognitive function of our participants. These included uncorrected (based on age) scores of NIH Fluid Composite ${ }^{50-52}$, NIH Flanker ${ }^{53,54}$ and NIH card sort ${ }^{53,55,56}$. A full explanation of these cognitive measures is available here ${ }^{37,57}$. These NIH toolboxes are highly validated. These measures were treated as a continuous measure. For all these variables, a higher score indicated higher cognitive performance.

\section{Covariates}

Family Structure. Family structure was a dichotomous variable: married $=1$ and not- married $=0$.

Parental Educational Attainment. Parental education was defined as a five-level categorical variable: (1) less than high school (reference category), (2) high school / GED, (3) some college, (4) college degree, and (5) graduate+ school.

Household Income. Household income was a nominal variable: Parents were asked, "What is your total combined highest income for the past 12 months? This should include income (before taxes and deductions) from all sources, wages, rent from properties, social security, disability and veteran's benefits, or unemployment benefits". Responses included less than $\$ 50,000, \$ 50,000-\$ 100,000$; and $\$ 100,000+$.

Race. Race was identified by the parents. Race was a categorical variable: Black, Asian, Other/Mixed, and White (reference group).

Ethnicity. Ethnicity was identified by the parents. Ethnicity was a categorical variable: 1 for Hispanic and 0 for Non-Hispanic (reference group).

Age. Age was a continuous measure in months and was calculated based on the birthdate reported by the child's parents.

\section{Moderator}

Sex. The sex of the child was a categorical variable with 1 for males and 0 for females.

\section{Analysis}

The program Data Analysis and Exploration Portal (DEAP) was utilized for statistical analyses. DEAP is an online analytical tool and is written on the R program. For our bivariate analysis, we used Chi-square and independent t-test to compare males and females for study variables. For our multivariable analysis, we used mixed-effect models because observations were nested to individuals, which were nested to families, and then to sites. As a result, we needed to have a random effect and fixed terms to adjust for the samples' clustering and non-independence. We ran two mixed-effects model regressions for each pair of independent and dependent model. All mixed-effects regression models were estimated in the overall/pooled sample. Model 1, the main effect model, was estimated in the absence of the sex by ADHD interaction term. Model 2 added interaction terms between sex and ADHD. BMI, cognitive function measures, ADHD symptoms, internalizing behavioral problem, externalizing behavioral problem, and total behavioral problem were the outcomes. Sex was the moderator. Screening for ADHD and history of diagnosis, both dichotomous variables, were the predictors. Our models controlled for age, race, ethnicity, parental education, household income, and family structure. Regression coefficient (b), standard error (SE), and $\mathrm{p}$-values were reported for each parameter.

\section{Ethics}

This secondary analysis used a fully de-identified data set. Our analysis was exempt from a full review of the Institutional Review Board (IRB). However, the original ABCD study protocol was approved by the IRB at the University of California, San Diego (UCSD). Children provided assent and parents provided consent ${ }^{38}$.

\section{Results}

Table 1 described the sample overall and by sex. The current analysis was performed on 10,171, 9-10-year-old children, from which 5,298 (52.1\%) were male and 4,873 (47.9\%) were female. Prevalence of positive screening for ADHD. History of a diagnosis was almost two times higher in males than females (20\% vs $10 \%)$.

As shown in Table 2, for each outcome, overall, history of diagnosis was associated with lower BMI and higher inattention, internalizing, externalizing, and total behavioral. Sex altered the association between history of diagnosis and internalizing, externalizing, and total behavioral problems as well as cognitive function but not BMI or inattention. A history of diagnosis was more strongly associated with internalizing, externalizing, and total behavioral problems for males than females. In contrast, history of diagnosis was more strongly associated with a decline in cognitive performance (two of three 
Table 1. Baseline data overall and by sex.

\begin{tabular}{|c|c|c|c|c|}
\hline Level & Overall & Female & Male & $\mathbf{p}$ \\
\hline \multirow[t]{2}{*}{$\mathbf{N}$} & 10,171 & 4,873 & 5,298 & \\
\hline & Mean (SD) & Mean (SD) & Mean (SD) & \\
\hline Age (Months) & $118.94(7.47)$ & $118.76(7.45)$ & $119.11(7.49)$ & 0.018 \\
\hline Body Mass Index (BMI) & $18.65(3.91)$ & $18.72(3.99)$ & $18.58(3.84)$ & 0.061 \\
\hline NIH Fluid Composite Uncorrected & $91.98(10.52)$ & $92.42(10.37)$ & $91.57(10.64)$ & $<0.001$ \\
\hline NIH Flanker Uncorrected & $94.26(8.91)$ & $94.08(8.73)$ & $94.43(9.07)$ & 0.045 \\
\hline NIH Card Sort Uncorrected & $92.87(9.34)$ & $93.44(8.87)$ & $92.34(9.73)$ & $<0.001$ \\
\hline Internalization Problems & $48.13(10.51)$ & $47.96(10.47)$ & $48.29(10.55)$ & 0.120 \\
\hline Externalization Problems & $46.02(9.59)$ & $45.80(9.54)$ & $46.22(9.63)$ & 0.028 \\
\hline Total Behavioral Problems & $43.05(10.16)$ & $42.83(10.14)$ & $43.26(10.19)$ & 0.033 \\
\hline Attention Deficit Hyperactivity Disorder (ADHD) Positive Screening & $53.27(5.59)$ & $53.10(5.43)$ & $53.43(5.73)$ & 0.003 \\
\hline \multirow[t]{2}{*}{ ADHD Screening (0-1) } & $0.09(0.29)$ & $0.06(0.24)$ & $0.12(0.33)$ & $<0.001$ \\
\hline & $\mathrm{N}(\%)$ & $N(\%)$ & $N(\%)$ & \\
\hline \multicolumn{5}{|l|}{ Race } \\
\hline White & $6,777(66.6)$ & $3,188(65.4)$ & $3,589(67.7)$ & 0.084 \\
\hline Black & $1,448(14.2)$ & $726(14.9)$ & $722(13.6)$ & \\
\hline Asian & $228(2.2)$ & $116(2.4)$ & $112(2.1)$ & \\
\hline Other/Mixed & $1,718(16.9)$ & $843(17.3)$ & $875(16.5)$ & \\
\hline \multicolumn{5}{|l|}{ Hispanic } \\
\hline No & $8,249(81.1)$ & $3,961(81.3)$ & 4,288 (80.9) & 0.672 \\
\hline Yes & $1,922(18.9)$ & $912(18.7)$ & $1,010(19.1)$ & \\
\hline \multicolumn{5}{|l|}{ Married Family } \\
\hline No & $3,071(30.2)$ & $1,516(31.1)$ & $1,555(29.4)$ & 0.056 \\
\hline Yes & $7,100(69.8)$ & 3,357 (68.9) & $3,743(70.6)$ & \\
\hline \multicolumn{5}{|l|}{ Parental Education } \\
\hline < HS Diploma & $367(3.6)$ & $185(3.8)$ & $182(3.4)$ & 0.756 \\
\hline HS Diploma/GED & $824(8.1)$ & $390(8.0)$ & $434(8.2)$ & \\
\hline Some College & $2,602(25.6)$ & $1,237(25.4)$ & $1,365(25.8)$ & \\
\hline Bachelor & $2,714(26.7)$ & $1,286(26.4)$ & $1,428(27.0)$ & \\
\hline Post Graduate Degree & $3,664(36.0)$ & $1,775(36.4)$ & 1,889 (35.7) & \\
\hline \multicolumn{5}{|l|}{ Household Income } \\
\hline$<50 K$ & $2,885(28.4)$ & $1,406(28.9)$ & 1,479 (27.9) & 0.510 \\
\hline$>=50 \mathrm{~K} \&<100 \mathrm{~K}$ & $2,916(28.7)$ & $1,398(28.7)$ & $1,518(28.7)$ & \\
\hline$>=100 \mathrm{~K}$ & $4,370(43.0)$ & $2,069(42.5)$ & $2,301(43.4)$ & \\
\hline \multicolumn{5}{|l|}{ History of Diagnosis } \\
\hline No & $8,683(85.4)$ & $4,384(90.0)$ & $4,299(81.1)$ & $<0.001$ \\
\hline Yes & $1,488(14.6)$ & $489(10.0)$ & $999(18.9)$ & \\
\hline
\end{tabular}

Table 2. Summary of our regressions without and with interactions on the associations between history of diagnosed Attention Deficit and Hyperactive Disorder (ADHD) and children internalizing, externalizing, and total behavioral problems ( $n=10171)$.

\begin{tabular}{|c|c|c|c|c|c|c|c|c|}
\hline & b & SE & $\mathrm{p}$ & Sig & b & SE & $\mathrm{p}$ & sig \\
\hline \multicolumn{9}{|l|}{ NIH Fluid Comp Uncorrected } \\
\hline Diagnosis History & -3.11506 & 0.26596 & $<0.001$ & $* * *$ & -3.97104 & 0.44220 & $<0.001$ & $* * *$ \\
\hline Sex (Male) & -0.76154 & 0.18832 & $<0.001$ & $* * *$ & -0.93662 & 0.20168 & $<0.001$ & $* * *$ \\
\hline Diagnosis History x Sex (Male) & & & & & 1.32645 & 0.54743 & 0.0154077 & $*$ \\
\hline \multicolumn{9}{|l|}{ NIH Card Sort Uncorrected } \\
\hline Diagnosis History & -1.75385 & 0.25202 & $<1 \mathrm{e}-6$ & $* * *$ & -2.75363 & 0.42094 & $<0.001$ & $* * *$ \\
\hline Sex (Male) & -1.10781 & 0.17778 & $<1 \mathrm{e}-6$ & $* * *$ & -1.31146 & 0.19051 & $<0.001$ & $* * *$ \\
\hline Diagnosis History $x$ Sex (Male) & & & & & 1.54554 & 0.52114 & 0.0030272 & $* *$ \\
\hline \multicolumn{9}{|l|}{ NIH Flanker } \\
\hline Diagnosis History & -2.41885 & 0.24296 & $<0.001$ & $* * *$ & -2.59236 & 0.40615 & $<0.001$ & $* * *$ \\
\hline Sex (Male) & 0.50576 & 0.17133 & 0.0031651 & $* *$ & 0.47050 & 0.18369 & 0.0104403 & $*$ \\
\hline Diagnosis History x Sex (Male) & & & & & 0.26813 & 0.50283 & 0.5938831 & \\
\hline
\end{tabular}




\begin{tabular}{|c|c|c|c|c|c|c|c|c|}
\hline \multicolumn{9}{|l|}{ Internalizing Symptoms } \\
\hline Diagnosis History & 1.06198 & 0.16659 & $<0.001$ & $* * *$ & 0.67024 & 0.25674 & 0.0090527 & $* *$ \\
\hline Sex (Male) & 0.00377 & 0.12488 & 0.9759113 & & -0.07887 & 0.13149 & 0.5486565 & \\
\hline Diagnosis History x Sex (Male) & & & & & 0.63321 & 0.31596 & 0.0450864 & $*$ \\
\hline \multicolumn{9}{|l|}{ Externalizing Symptoms } \\
\hline Diagnosis History & 0.71445 & 0.15362 & 0.001 & $* * *$ & 0.32813 & 0.23673 & 0.1657568 & \\
\hline Sex (Male) & -0.06859 & 0.11520 & 0.5515747 & & -0.15014 & 0.12130 & 0.2158118 & \\
\hline Diagnosis History x Sex (Male) & & & & & 0.62494 & 0.29134 & 0.0319724 & $*$ \\
\hline \multicolumn{9}{|l|}{ Total } \\
\hline Diagnosis History & 0.92653 & 0.14863 & $<0.001$ & $* * *$ & 0.55601 & 0.22794 & 0.0147341 & $*$ \\
\hline Sex (Male) & -0.09435 & 0.11187 & 0.3990413 & & -0.17269 & 0.11767 & 0.1422296 & \\
\hline Diagnosis History x Sex (Male) & & & & & 0.60085 & 0.28045 & 0.0321783 & $*$ \\
\hline \multicolumn{9}{|l|}{ ADHD Symptoms } \\
\hline Diagnosis History & 0.57593 & 0.08872 & $<0.001$ & $* * *$ & 0.46201 & 0.13675 & 0.0007311 & $* * *$ \\
\hline Sex (Male) & 0.02577 & 0.06654 & 0.6985967 & & 0.00166 & 0.07009 & 0.9811147 & \\
\hline Diagnosis History x Sex (Male) & & & & & 0.18504 & 0.16828 & 0.2715564 & \\
\hline \multicolumn{9}{|l|}{ Body Mass Index (BMI) } \\
\hline Diagnosis History & -0.52673 & 0.10250 & $<0.001$ & $* * *$ & -0.40763 & 0.16942 & 0.0161422 & $*$ \\
\hline Sex (Male) & -0.11892 & 0.07286 & 0.102696 & & -0.09454 & 0.07790 & 0.2249698 & \\
\hline Diagnosis History $x$ Sex (Male) & & & & & -0.18494 & 0.20945 & 0.3772561 & \\
\hline
\end{tabular}

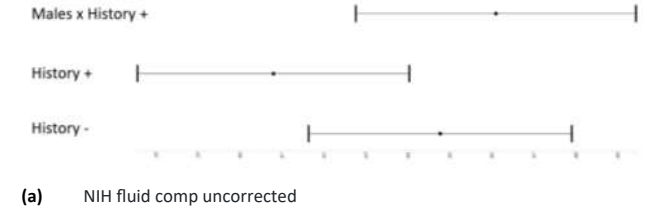

(a) NIH fluid comp uncorrected

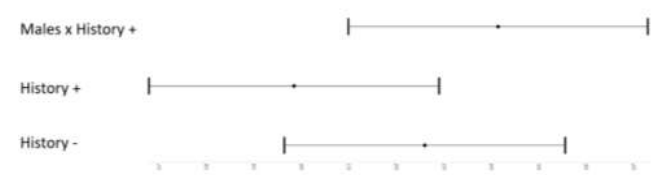

(b) NIH card sort uncorrected

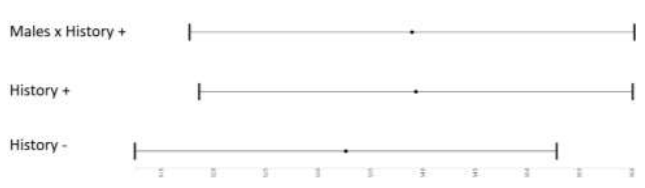

(c) Internalizing problems

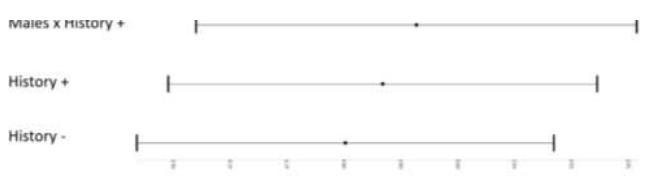

(d) Externalizing problems

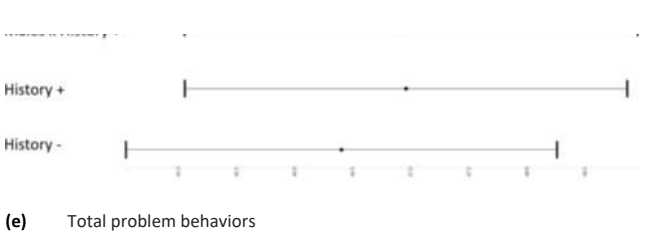

Figure 1. Associations between history of diagnosed Attention Deficit and Hyperactive Disorder (ADHD) and children internalizing, externalizing, total behavioral problems, and cognitive function by $\operatorname{sex}(n=10171)$. measures) for females than in males. The association between history of diagnosis and ADHD symptoms, BMI, or Flanker measure (cognition) did not differ between males and females (Figure 1).

Table 2. Summary of our regressions without and with interactions on the associations between history of diagnosed Attention Deficit and Hyperactive Disorder (ADHD) and children internalizing, externalizing, and total behavioral problems $(\mathrm{n}=10171)$.

When we tested the same sex/gender differences for positive ADHD screening, we did not find most of the sex/ gender interactions that were significant for history of diagnosis, which were shown in Table 2. We only found two significant interactions between sex/gender and positive screening for ADHD on externalizing and total problems, suggesting that the associations between positive ADHD screening and externalizing and total problems are stronger for males than females. There were no significant interactions between sex/gender and positive screening for ADHD on other cognitive or emotional outcomes (Table 3 and Figure 2).

\section{Discussion}

In a national sample of 9-10-year-old children in the US, while history and positive screening for ADHD were both associated with higher internalizing, externalizing, and total behavioral problems as well as lower cognitive performance, sexes showed more differences in emotional, behavioral, and cognitive correlates of history of diagnosis than positive screening for ADHD. The observation of stronger sex differences in behavioral, emotional, and cognitive correlates for history of diagnosis than positive 
Table 3. Summary of our regressions without and with interactions on the association between positive screening for Attention Deficit and Hyperactive Disorder (ADHD) and children internalizing, externalizing, and total behavioral problems ( $n=10171$ ).

\begin{tabular}{|c|c|c|c|c|c|c|c|c|}
\hline & b & SE & $\mathbf{p}$ & Sig & b & SE & $\mathbf{p}$ & sig \\
\hline \multicolumn{9}{|l|}{ NIH Fluid Comp Uncorrected } \\
\hline Positive Screening & -2.95823 & 0.32211 & $<1 \mathrm{e}-6$ & $* * *$ & -3.48091 & 0.55660 & $<1 \mathrm{e}-6$ & $* * *$ \\
\hline Sex (Male) & -0.85829 & 0.18930 & $5.9 e-06$ & $* * *$ & -0.92231 & 0.19728 & $3 e-06$ & $* * *$ \\
\hline Positive Screening x Sex (Male) & & & & & 0.78174 & 0.67912 & 0.2497188 & \\
\hline \multicolumn{9}{|l|}{ NIH Card Sort Uncorrected } \\
\hline Positive Screening & -1.79677 & 0.30497 & $<1 \mathrm{e}-6$ & $* * *$ & -1.81167 & 0.52797 & 0.0006029 & $* * *$ \\
\hline Sex (Male) & -1.16187 & 0.17815 & $<1 \mathrm{e}-6$ & $* * *$ & -1.16368 & 0.18576 & $<1 \mathrm{e}-6$ & $* * *$ \\
\hline Positive Screening x Sex (Male) & & & & & 0.02228 & 0.64447 & 0.9724238 & \\
\hline \multicolumn{9}{|l|}{ NIH Flanker } \\
\hline Positive Screening & -1.58365 & 0.29496 & $<1 \mathrm{e}-6$ & $* * *$ & -2.10565 & 0.51071 & $3.77 e-05$ & $* * *$ \\
\hline Sex (Male) & 0.42990 & 0.17232 & 0.0126184 & $*$ & 0.36602 & 0.17970 & 0.0416875 & $*$ \\
\hline Positive Screening x Sex (Male) & & & & & 0.78065 & 0.62346 & 0.210557 & \\
\hline Positive Screening & 1.85149 & 0.19486 & $<1 \mathrm{e}-6$ & $* * *$ & 1.68605 & 0.31357 & $<1 \mathrm{e}-6$ & $* * *$ \\
\hline Sex (Male) & -0.02514 & 0.12599 & 0.8418402 & & -0.04632 & 0.12983 & 0.7212548 & \\
\hline Positive Screening x Sex (Male) & & & & & 0.26344 & 0.38918 & 0.4984707 & \\
\hline \multicolumn{9}{|l|}{ Externalizing Symptoms } \\
\hline Positive Screening & 1.58559 & 0.17895 & $<1 \mathrm{e}-6$ & $* * *$ & 0.61816 & 0.28652 & 0.0309909 & $*$ \\
\hline Sex (Male) & -0.13443 & 0.11578 & 0.2456099 & & -0.25663 & 0.11882 & 0.0308085 & $*$ \\
\hline Positive Screening x Sex (Male) & & & & & 1.51528 & 0.35567 & $2.06 \mathrm{e}-05$ & $* * *$ \\
\hline \multicolumn{9}{|l|}{ Total } \\
\hline Positive Screening & 1.76565 & 0.17304 & $<1 \mathrm{e}-6$ & $* * *$ & 1.30080 & 0.27693 & $2.7 e-06$ & $* * *$ \\
\hline Sex (Male) & -0.13565 & 0.11259 & 0.2282954 & & -0.19477 & 0.11587 & 0.0928 & $\cdot$ \\
\hline Positive Screening x Sex (Male) & & & & & 0.73794 & 0.34416 & 0.0320438 & $*$ \\
\hline \multicolumn{9}{|l|}{ ADHD Symptoms } \\
\hline Positive Screening & 1.16274 & 0.10416 & $<1 \mathrm{e}-6$ & $* * *$ & 0.94217 & 0.16757 & $<1 \mathrm{e}-6$ & $* * *$ \\
\hline Positive Screening x Sex (Male) & & & & & 0.35020 & 0.20798 & 0.0922458 & . \\
\hline \multicolumn{9}{|l|}{ Body Mass Index (BMI) } \\
\hline Positive Screening & -0.40187 & 0.20952 & 0.055177 & . & -0.38772 & 0.38244 & 0.3107365 & \\
\hline Sex (Male) & -0.11692 & 0.12249 & 0.3398491 & & -0.11547 & 0.12677 & 0.3624215 & \\
\hline Positive Screening x Sex (Male) & & & & & -0.02013 & 0.45403 & 0.9646434 & \\
\hline
\end{tabular}

screening for ADHD suggests that at least some of the malefemale differences in cognitive, emotional and behavioral correlates of ADHD in clinical samples are possibly due to differential referral (gender differences) rather than biological differences (sex differences). This suggests that differential manifestation of ADHD for boys and girls is more prominent in clinical than community settings.

The current study compared males and females for cognitive, emotional, and behavioral problems that are commonly found in children with ADHD. These problems include difficulty paying attention, difficulty controlling impulsive behaviors, overactivity ${ }^{2,8,9}$, internalization ${ }^{16,58-61}$, and externalization to name a few. Some of these features are core elements of ADHD (e.g., attention problem) and some others (e.g., internalizing behaviors) are believed to be comorbid to it. For example, lower performance in cognitive domains, particularly executive function ${ }^{6}$ and impulse control ${ }^{7,8}$, are core to ADHD, while high levels of internalizing $^{9-11}$, externalizing ${ }^{11-14}$, and total behavioral problems $^{15,16}$ is believed to be a common comorbidity.

In line with our findings, literature has shown that particularly in clinical settings, males and females differ in ADHD presentation ${ }^{8,24,28-30}$. Sex differences in the association between ADHD and depression, impulsivity, cognitive function, and problem behaviors are consistently reported ${ }^{24-26,31,32}$., Policy and clinical implications of these observations depend on whether they are due to gender differences (social processes) such as differential referral of boys and girls, or sex differences (due to biology) that are innate and non-modifiable ${ }^{31}$.

Sex differences in ADHD is not limited to the prevalence ${ }^{18-21}$ as males and females show different ADHD correlates $^{22-27}$. In line with our findings on correlates of history of diagnosis, a meta-analysis by Gersgon 


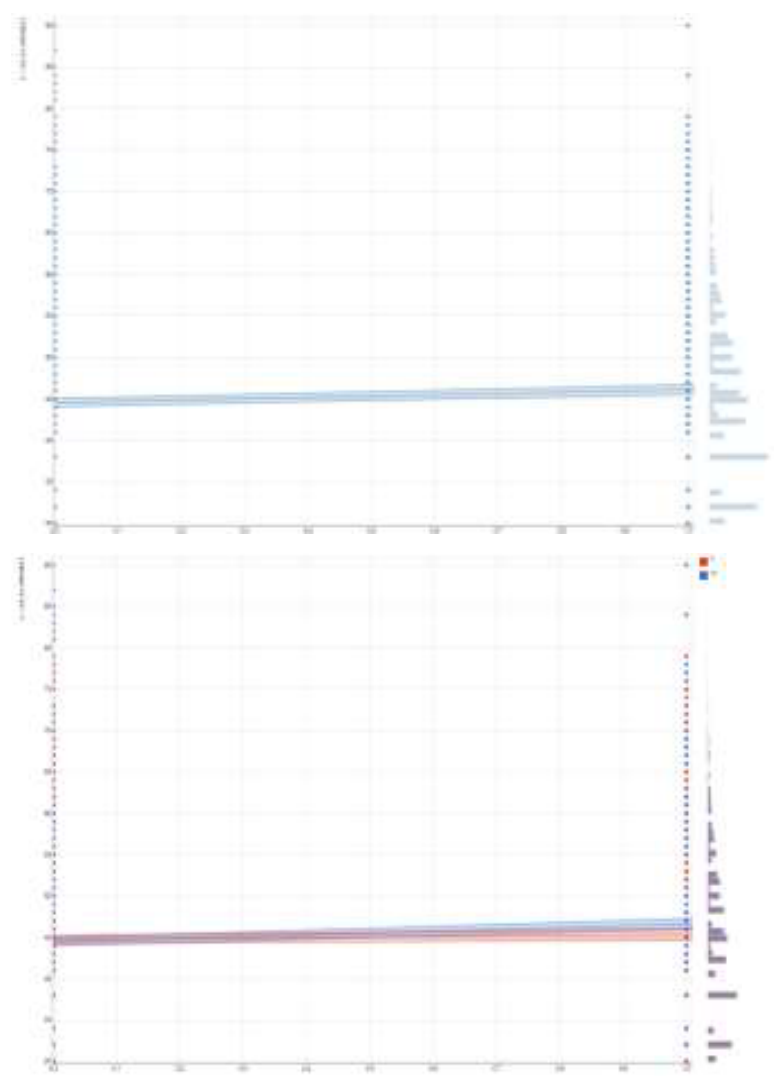

External

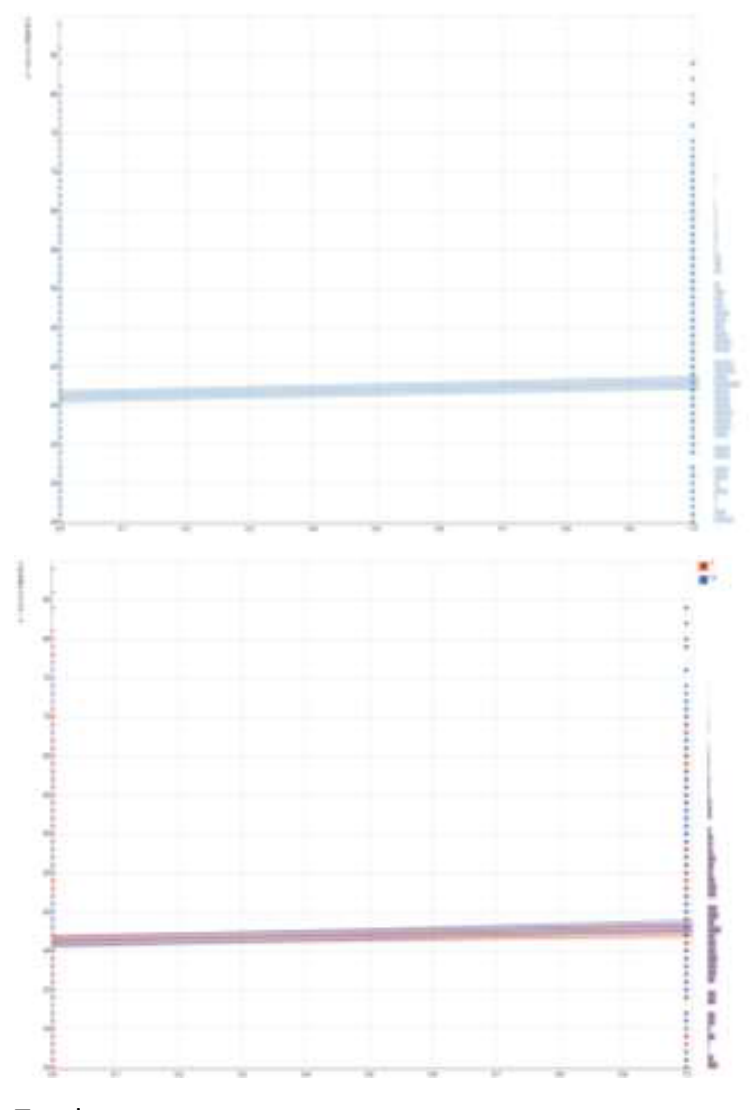

Total

Figure 2. Association between positive screening for Attention Deficit and Hyperactive Disorder (ADHD) and children externalizing and total behavioral problems by sex $(n=10171)$.

documented greater intellectual impairment in girls than boys with $\mathrm{ADHD}^{31}$. In line with our findings on correlates of history of diagnosis, the Gersgon meta-analysis showed higher externalizing problems in boys than girls with $\mathrm{ADHD}^{31}$. Our results on correlates of history of diagnosis are also in line with the meta-analysis by Gaub and Carlson, showing that compared with ADHD boys, ADHD girls may display greater intellectual impairment and lower rates of other externalizing behaviors ${ }^{17}$.

However, we did not find many of male-female differences for correlates of positive screening for ADHD. The results of the previous original articles and metaanalyses are also not always consistent, as the literature is full of original studies that have reported opposite results regarding sex and gender differences in correlates of ADHD. In contrast to our finding, the Gersgon meta-analysis and Gaub and Carlson showed lower hyperactivity, inattention, impulsivity in girls than boys with ADHD.

Our finding on sex and gender differences were more pronounced when we compared boys and females for a diagnosis history rather than a positive screening. This observation is in support of the argument by Gaub and Carlson who suggested that differential referral and paths to diagnosis may explain some of the gender differences in ADHD burden and presentation. As they are preventable and due to social rather than biological mechanisms, they can be considered disparities and should be prevented. In this view, some of the differences in the burden of ADHD between males and females is due to differences in the referral bias for girls and boys ${ }^{17}$. As such, in research on sex and gender differences in ADHD, there is a need to adjust for history of diagnosis and referral source. Similarly, the results in clinical and community-settings would not necessarily agree. In other words, similar differences can be replicated in children with ADHD who are not referred and screened ${ }^{17}$.

Gaub and Carlson mentioned that girls with ADHD displayed lower levels of inattention, internalizing behavior, and peer aggression than boys with $\mathrm{ADHD}^{17}$. In clinicreferred samples, however, boys and girls with ADHD may display more similar impairment on emotional, behavioral, and cognitive domains ${ }^{17}$. Other than referral bias, some of the sex and gender differences in correlates of ADHD may be due to comorbidity, developmental patterns, and rater source ${ }^{17}$.

As Gaub and Carlson and others have argued, we also 
believe that sources of gender and sex differences in the correlates and burden of ADHD require more research ${ }^{17}$. We may expect more gender differences in correlates of ADHD in the literature that has sampled males and females from clinics. In the $A B C D$ sample, there are fewer differences between correlates of positive ADHD screening for males and females. As ABCD has collected extensive brain image data, it has provided an excellent opportunity to test sex differences in correlates of ADHD. Further analysis of the $A B C D$ data can help decompose sex and gender differences in ADHD by using functional, structural, and diffusion MRI data.

Both sex and gender differences can explain why males and females with ADHD have different levels of internalizing, externalizing, total behavioral problems, and cognitive performance. Sex refers to the biology of being male or female. For instance, a wide range of studies has documented structural and functional brain differences in ADHD in males and females. In contrast to sex as a biological factor, gender may also explain some of the observed differences in this study. Some of the sex differences in brain and behavior are due to chromosomal differences of males and females.Sex differences are also not preventable and can be seen as disparities and inequalities that should or could be potentially eliminated, as they are fixed and inherent. As a result of gender, a social construct, socialization and experiences of boys and girls with ADHD differ. Such differences include parenting, social norms, socialization, expectations, and behavioral coping, which may also differ between boys and girls with and without ADHD. In contrast to sex differences that are not the target of policy, gender differences might be preventable and to some levels can be seen as disparities and inequalities between males and females that are shaped by social forces. Such gender differences in the burden of ADHD of boys and girls can be potentially eliminated, however, we do not argue that they are easy to change.

\section{Implications}

Our study may have some implications for research and practice. Clinicians should be aware that among individuals with a history of diagnosis, ADHD may be associated with worse externalizing behaviors for males and worse cognitive problems for females. This information may also help health care providers and parents to tailor clinical interventions to the sex/gender of their child. As the presentation of boys and girls with a history of diagnosed ADHD differs, the treatment approach may consider such existing sex/gender differences. As such, interventions and programs in clinical settings may benefit from tailoring our modalities to the sex/gender of the ADHD patients who have received a diagnosis. Our result reminds researchers that sex/gender is more than a control variable when we work with children with a history of diagnosed ADHD.
Policymakers should be aware that some of the sex differences in cognitive, behavioral, and emotional needs of 9-10-year-old children with diagnosed ADHD may be an artifact of diagnosis and referral rather than a sex-specific presentation of ADHD.

\section{Future research}

As this study was preliminary, more research is needed in the future. More research is needed to decompose the effect of biology (sex) from society (gender) in explaining differences in the burden of ADHD for boys and girls. We need brain imaging studies that explore differential implication of cortical and sub-cortical regions for males and females with ADHD. Similarly, we need studies that explore parent and teacher expectations and practices with males and females with ADHD.

\section{Limitations}

Our study has some limitations. One major limitation was that we did not account for family history, other psychiatric disorders, duration of the illness, or history of treatment. We also did not measure the severity of ADHD other than an over-simplistic measure of inattention. Additionally, for ADHD symptoms, inattention, internalizing and externalizing disorders, we only relied on parental reports. Inclusion of other sources such as the child, teacher, and medical charts, and school record could increase our ability to observe differences from other viewpoints. We did not validate our results based on the electronic records of the individuals or school charts. We also did not include any family, social, or brain imaging data that could potentially explain some of the observed sex and gender differences. Finally, we did not measure other aspects of physical health rather than BMI.

\section{Future research}

There is a need to study how sex, a biological construct, and gender, a social construct, shape differential correlates and burden of ADHD in males and females. As one size does not fit all, sex and gender differences themselves may differ across settings. There is a need to study social and biological processes that may explain male-female differences in correlates of ADHD. Differences in referral as well as norms, expectations, coping, and parenting may explain some of the differences in the behaviors of males and females with ADHD. Sex hormones, sex chromosomes, puberty, and brain structures and functions may also be involved in explaining why boys and girls who have ADHD have a different cognitive, behavioral, and emotional profile.

\section{Conclusions}

In 9-10-year-old children in the US, sex and gender may alter how ADHD is correlated with cognitive, behavioral, 
and emotional problems. While the history of diagnosis is more strongly associated with externalizing behavioral problems for males than in females, females with a history of diagnosis experience more significant problems in cognitive performance than their male counterparts. These gender differences are less pronounced when we compare males and females who screen positive for ADHD, suggesting that differential referral and chance of diagnosis may contribute to some of the gender differences. If this hypothesis is confirmed by future research, some of the male-female differences in correlates of ADHD might be regarded as preventable.

\section{Conflicts of Interest}

Author declares no conflicts of interest.

\section{Author Funding}

SA is supported by the following National Institutes of Health (NIH) grants: CA201415 02, DA035811-05, U54MD007598, U54MD008149, D084526-03, and U54CA229974.

\section{DEAP Funding}

DEAP is a software provided by the Data Analysis and Informatics Center of ABCD located at the UC San Diego with generous support from the National Institutes of Health and the Centers for Disease Control and Prevention under award number U24DA041123. The DEAP project information and links to its source code are available under the resource identifier RRID: SCR_016158.

\section{ABCD Acknowledgments}

Data used in the preparation of this article were obtained from the Adolescent Brain Cognitive Development (ABCD) Study (https://abcdstudy.org), held in the NIMH Data Archive (NDA). This is a multisite, longitudinal study designed to recruit more than 10,000 children age 9-10 and follow them over 10 years into early adulthood. The ABCD Study is supported by the National Institutes of Health Grants [U01DA041022, U01DA041028, U01DA041048, U01DA041089, U01DA041106, U01DA041117, U01DA041120, U01DA041134, U01DA041148, U01DA041156, U01DA041174, U24DA041123, U24DA041147]. A full list of supporters is available at https://abcdstudy.org/nih-collaborators. A listing of participating sites and a complete listing of the study investigators can be found at https://abcdstudy. org/principal-investigators.html. ABCD consortium investigators designed and implemented the study and/or provided data but did not necessarily participate in analysis or writing of this report. This manuscript reflects the views of the authors and may not reflect the opinions or views of the $\mathrm{NIH}$ or $\mathrm{ABCD}$ consortium investigators. The ABCD data repository grows and changes over time. The $A B C D$ data used in this report came from [NIMH Data Archive Digital Object Identifier (http://dx.doi.org/10.15154/1504041)].

\section{Acknowledgment}

Authors wished to thank Luke Sorensen for his edits to this paper.

\section{Conflicts of Interest}

The funders had no role in the design of the study; in the collection, analyses, or interpretation of data; in the writing of the manuscript, or in the decision to publish the results.

\section{References}

1. Faraone SV, Sergeant J, Gillberg C, Biederman J. The worldwide prevalence of ADHD: is it an American condition? World Psychiatry. 2003;2(2):104-113.

2. Polanczyk G, de Lima MS, Horta BL, Biederman J, Rohde LA. The worldwide prevalence of ADHD: a systematic review and metaregression analysis. Am J Psychiatry. 2007;164(6):942-948.

3. Ji Y, Hong X, Wang G, et al. A Prospective Birth Cohort Study on Early Childhood Lead Levels and Attention Deficit Hyperactivity Disorder: New Insight on Sex Differences. J Pediatr. 2018;199:124-131 e128.

4. Smidts DP, Oosterlaan J. How common are symptoms of ADHD in typically developing preschoolers? A study on prevalence rates and prenatal/demographic risk factors. Cortex. 2007;43(6):710-717.

5. Doyle LR, Glass L, Wozniak JR, et al. Relation Between Oppositional/ Conduct Behaviors and Executive Function Among Youth with Histories of Heavy Prenatal Alcohol Exposure. Alcohol Clin Exp Res. 2019;43(6):1135-1144.

6. Martel MM. Individual differences in attention deficit hyperactivity disorder symptoms and associated executive dysfunction and traits: sex, ethnicity, and family income. Am J Orthopsychiatry. 2013;83(2 Pt 3):165-175.

7. Newcorn JH, Halperin JM, Jensen PS, et al. Symptom profiles in children with ADHD: effects of comorbidity and gender. J Am Acad Child Adolesc Psychiatry. 2001;40(2):137-146.

8. Welkie J, Babinski DE, Neely KA. Sex and Emotion Regulation Difficulties Contribute to Depression in Young Adults With AttentionDeficit/Hyperactivity Disorder. Psychol Rep. 2020:33294120918803.

9. Noren Selinus E, Molero Y, Lichtenstein P, et al. Subthreshold and threshold attention deficit hyperactivity disorder symptoms in childhood: psychosocial outcomes in adolescence in boys and girls. Acta Psychiatr Scand. 2016;134(6):533-545.

10. Babinski DE, Waschbusch DA, Waxmonsky JG. Sex and Pubertal Status Moderate the Association Between ADHD and Depression Symptoms: An Examination From Preadolescence Through Late Adolescence. J Clin Psychiatry. 2019;80(3).

11. Hamzeloo M, Mashhadi A, Salehi Fadardi J. The Prevalence of ADHD and Comorbid Disorders in Iranian Adult Male Prison Inmates. J Atten Disord. 2016;20(7):590-598.

12. Bron TI, Bijlenga D, Verduijn J, Penninx BW, Beekman AT, Kooij JJ. Prevalence of ADHD symptoms across clinical stages of major depressive disorder. J Affect Disord. 2016;197:29-35.

13. Val-Laillet D, Aarts E, Weber B, et al. Neuroimaging and neuromodulation approaches to study eating behavior and prevent and treat eating disorders and obesity. Neuroimage Clin. 2015;8:1-31.

14. Biederman J, Mick E, Faraone SV, et al. Influence of gender on attention deficit hyperactivity disorder in children referred to a psychiatric clinic. Am J Psychiatry. 2002;159(1):36-42. 
15. Park JI, Shim SH, Lee M, et al. The validities and efficiencies of korean ADHD rating scale and korean child behavior checklist for screening children with ADHD in the community. Psychiatry Investig. 2014;11(3):258-265.

16. Slobodin O, Davidovitch M. Gender Differences in Objective and Subjective Measures of ADHD Among Clinic-Referred Children. Front Hum Neurosci. 2019;13:441.

17. Gaub M, Carlson CL. Gender differences in ADHD: a meta-analysis and critical review. J Am Acad Child Adolesc Psychiatry. 1997;36(8):10361045.

18. Derks EM, Hudziak JJ, Boomsma DI. Why more boys than girls with ADHD receive treatment: a study of Dutch twins. Twin Res Hum Genet. 2007;10(5):765-770.

19. Schote AB, C ALS, Pabst K, Meier JK, Frings C, Meyer J. Sex, ADHD symptoms, and CHRNA5 genotype influence reaction time but not response inhibition. J Neurosci Res. 2019;97(2):215-224.

20. Meyer BJ, Stevenson J, Sonuga-Barke EJS. Sex Differences in the Meaning of Parent and Teacher Ratings of ADHD Behaviors: An Observational Study. J Atten Disord. 2020;24(13):1847-1856.

21. Bitter I, Simon V, Balint S, Meszaros A, Czobor P. How do different diagnostic criteria, age and gender affect the prevalence of attention deficit hyperactivity disorder in adults? An epidemiological study in a Hungarian community sample. Eur Arch Psychiatry Clin Neurosci. 2010;260(4):287-296.

22. Monuteaux MC, Mick E, Faraone SV, Biederman J. The influence of sex on the course and psychiatric correlates of ADHD from childhood to adolescence: a longitudinal study. J Child Psychol Psychiatry. 2010;51(3):233-241.

23. Seidman LJ, Biederman J, Monuteaux MC, Valera E, Doyle AE, Faraone SV. Impact of gender and age on executive functioning: do girls and boys with and without attention deficit hyperactivity disorder differ neuropsychologically in preteen and teenage years? Dev Neuropsychol. 2005;27(1):79-105.

24. Carlson CL, Tamm L, Gaub M. Gender differences in children with ADHD, ODD, and co-occurring ADHD/ODD identified in a school population. J Am Acad Child Adolesc Psychiatry. 1997;36(12):17061714.

25. Williamson D, Johnston C. Gender differences in adults with attentiondeficit/hyperactivity disorder: A narrative review. Clin Psychol Rev. 2015;40:15-27.

26. Cortese S, Faraone SV, Bernardi S, Wang S, Blanco C. Gender differences in adult attention-deficit/hyperactivity disorder: results from the National Epidemiologic Survey on Alcohol and Related Conditions (NESARC). J Clin Psychiatry. 2016;77(4):e421-428.

27. Ohan JL, Johnston C. Gender appropriateness of symptom criteria for attention-deficit/hyperactivity disorder, oppositionaldefiant disorder, and conduct disorder. Child Psychiatry Hum Dev. 2005;35(4):359-381.

28. Freund N, MacGillivilray HT, Thompson BS, et al. Sex-dependent changes in ADHD-like behaviors in juvenile rats following cortical dopamine depletion. Behav Brain Res. 2014;270:357-363.

29. Valera EM, Brown A, Biederman J, et al. Sex differences in the functional neuroanatomy of working memory in adults with ADHD. Am J Psychiatry. 2010;167(1):86-94.

30. Munoz-Suazo MD, Navarro-Munoz J, Diaz-Roman A, Porcel-Galvez AM, Gil-Garcia E. Sex differences in neuropsychological functioning among children with attention-deficit/hyperactivity disorder. Psychiatry Res. 2019;278:289-293.

31. Gershon J. A meta-analytic review of gender differences in ADHD. $J$ Atten Disord. 2002;5(3):143-154.

32. Jahangard L, Haghighi M, Bajoghli H, Holsboer-Trachsler E, Brand
S. Among a sample of Iranian students, adult attention deficit hyperactivity disorder is related to childhood ADHD, but not to age, gender, socioeconomic status, or birth order--an exploratory study. Int J Psychiatry Clin Pract. 2013;17(4):273-278.

33. Alcohol Research: Current Reviews Editorial S. NIH's Adolescent Brain Cognitive Development (ABCD) Study. Alcohol Res. 2018;39(1):97.

34. Casey BJ, Cannonier T, Conley MI, et al. The Adolescent Brain Cognitive Development (ABCD) study: Imaging acquisition across 21 sites. Dev Cogn Neurosci. 2018;32:43-54.

35. Karcher NR, O’Brien KJ, Kandala S, Barch DM. Resting-State Functional Connectivity and Psychotic-like Experiences in Childhood: Results From the Adolescent Brain Cognitive Development Study. Biol Psychiatry. 2019;86(1):7-15.

36. Lisdahl KM, Sher KJ, Conway KP, et al. Adolescent brain cognitive development (ABCD) study: Overview of substance use assessment methods. Dev Cogn Neurosci. 2018;32:80-96.

37. Luciana M, Bjork JM, Nagel BJ, et al. Adolescent neurocognitive developmentand impacts of substance use: Overview of the adolescent brain cognitive development (ABCD) baseline neurocognition battery. Dev Cogn Neurosci. 2018;32:67-79.

38. Auchter AM, Hernandez Mejia M, Heyser CJ, et al. A description of the ABCD organizational structure and communication framework. Dev Cogn Neurosci. 2018;32:8-15.

39. Penelo E, De la Osa N, Navarro JB, Domènech JM, Ezpeleta L. The Brief Problem Monitor-Parent form (BPM-P), a short version of the Child Behavior Checklist: Psychometric properties in Spanish 6-to 8-yearold children. Psychological assessment. 2017;29(11):1309.

40. Daniels B, Volpe RJ, Fabiano GA, Briesch AM. Classification accuracy and acceptability of the Integrated Screening and Intervention System Teacher Rating Form. School Psychology Quarterly. 2017;32(2):212.

41. Achenbach T, McConaughy S, Ivanova M, Rescorla L. Manual for the ASEBA brief problem monitor (BPM). Burlington, VT: ASEBA. 2011:133.

42. Piper BJ, Gray HM, Raber J, Birkett MA. Reliability and validity of Brief Problem Monitor, an abbreviated form of the Child Behavior Checklist. Psychiatry and clinical neurosciences. 2014;68(10):759-767.

43. Bilenberg N. The Child Behavior Checklist (CBCL) and related material: standardization and validation in Danish population based and clinically based samples. Acta Psychiatr Scand Suppl. 1999;398:252 .

44. Bordin IA, Rocha MM, Paula CS, et al. Child Behavior Checklist (CBCL),Youth Self-Report (YSR) and Teacher's Report Form(TRF): an overview of the development of the original and Brazilian versions. Cad Saude Publica. 2013;29(1):13-28.

45. Diler RS, Birmaher B, Axelson D, et al. The Child Behavior Checklist (CBCL) and the CBCL-bipolar phenotype are not useful in diagnosing pediatric bipolar disorder. J Child Adolesc Psychopharmacol. 2009;19(1):23-30.

46. Dominguez-Lara SA. [Evidences of predictive capability of Child Behavior Checklist 1.5-5 (CBCL/1.5-5)]. Rev Chil Pediatr. 2017;88(2):290-291.

47. Kaat AJ, Blackwell CK, Estabrook R, et al. Linking the Child Behavior Checklist (CBCL) with the Multidimensional Assessment Profile of Disruptive Behavior (MAP-DB): Advancing a dimensional spectrum approach to disruptive behavior. J Child Fam Stud. 2019;28(2):343353.

48. Kristensen S, Henriksen TB, Bilenberg N. The Child Behavior Checklist for Ages 1.5-5 (CBCL/1(1/2)-5): assessment and analysis of parentand caregiver-reported problems in a population-based sample of Danish preschool children. Nord J Psychiatry. 2010;64(3):203-209.

49. Papachristou E, Ormel J, Oldehinkel AJ, et al. Child Behavior 
Checklist-Mania Scale (CBCL-MS): development and evaluation of a population-based screening scale for bipolar disorder. PLoS One. 2013;8(8):e69459.

50. Heaton RK, Akshoomoff N, Tulsky D, et al. Reliability and validity of composite scores from the NIH Toolbox Cognition Battery in adults. Journal of the International Neuropsychological Society: JINS. 2014;20(6):588.

51. Bauer PJ, Zelazo PD. Ix. Nih toolbox cognition battery (cb): summary, conclusions, and implications for cognitive development. Monographs of the Society for Research in Child Development. 2013;78(4):133-146.

52. Akshoomoff N, Beaumont JL, Bauer PJ, et al. VIII. NIH Toolbox Cognition Battery (CB): composite scores of crystallized, fluid, and overall cognition. Monographs of the Society for Research in Child Development. 2013;78(4):119-132.

53. Zelazo PD, Anderson JE, Richler J, Wallner-Allen K, Beaumont JL, Weintraub S. II. NIH Toolbox Cognition Battery (CB): Measuring executive function and attention. Monographs of the Society for Research in Child Development. 2013;78(4):16-33.

54. Akshoomoff N, Newman E, Thompson WK, et al. The NIH Toolbox Cognition Battery: results from a large normative developmental sample (PING). Neuropsychology. 2014;28(1):1.

55. Weintraub S, Dikmen SS, Heaton RK, et al. The cognition battery of the NIH toolbox for assessment of neurological and behavioral function: validation in an adult sample. Journal of the International Neuropsychological Society: JINS. 2014;20(6):567.
56. Zelazo PD, Anderson JE, Richler J, et al. NIH Toolbox Cognition Battery (CB): validation of executive function measures in adults. Journal of the International Neuropsychological Society: JINS. 2014;20(6):620.

57. Thompson WK, Barch DM, Bjork JM, et al. The structure of cognition in 9 and 10 year-old children and associations with problem behaviors: Findings from the ABCD study's baseline neurocognitive battery. Dev Cogn Neurosci. 2019;36:100606.

58. Katsuki D, Yamashita H, Yamane K, Kanba S, Yoshida K. Clinical Subtypes in Children with Attention-Deficit Hyperactivity Disorder According to Their Child Behavior Checklist Profile. Child Psychiatry Hum Dev. 2020.

59. Donfrancesco R, Innocenzi M, Marano A, Biederman J. Deficient Emotional Self-Regulation in ADHD Assessed Using a Unique Profile of the Child Behavior Checklist (CBCL): Replication in an Italian Study. J Atten Disord. 2015;19(10):895-900.

60. Peyre H, Speranza M, Cortese S, Wohl M, Purper-Ouakil D. Do ADHD children with and without child behavior checklist-dysregulation profile have different clinical characteristics, cognitive features, and treatment outcomes? J Atten Disord. 2015;19(1):63-71.

61. Uchida M, Faraone SV, Martelon M, et al. Further evidence that severe scores in the aggression/anxiety-depression/attention subscales of child behavior checklist (severe dysregulation profile) can screen for bipolar disorder symptomatology: a conditional probability analysis. J Affect Disord. 2014;165:81-86. 


\section{Appendix 1. Model Formula}

nihtbx_flanker_uncorrected scrn_commondx + race.4level + hisp + sex + age + high.educ.bl + household.income.bl + married.bl

nihtbx_cardsort_uncorrected scrn_commondx + race.4level + hisp + sex + age + high.educ. bl + household.income.bl + married.bl

nihtbx_fluidcomp_uncorrected $\sim$ scrn_commondx + race.4level + hisp + sex + age + high.educ.bl + household.income.bl + married.bl

a

asr_scr_totprob_t $\sim$ scrn_commondx + race.4level + hisp + sex + age + high.educ.bl + household.income.bl + married.bl

asr_scr_internal_t ${ }^{\sim}$ scrn_commondx + race.4level + hisp + sex + age + high.educ. bl + household.income.bl + married.bl

asr_scr_external_t $\sim$ scrn_commondx + race.4level + hisp + sex + age + high.educ. bl + household.income.bl + married.bl

asr_scr_adhd_t scrn_commondx + race.4level + hisp + sex + age + high.educ.bl + household.income.bl + married.bl

anthro_bmi_calc ${ }^{\sim}$ scrn_commondx + race.4level + hisp + sex + age + high.educ.bl + household.income.bl + married.bl$$
+
$$

nihtbx_flanker_uncorrected $\sim$ scrn_commondx + race.4level + hisp + sex + age + high.educ.bl + household.income.bl + married.bl + scrn commondx $*$ sex

nihtbx_cardsort_uncorrected $\sim$ scrn_commondx + race.4level + hisp + sex + age + high.educ.bl + household.income.bl + married.bl + scrn commondx * sex

nihtbx_fluidcomp_uncorrected $\sim$ scrn_commondx + race.4level + hisp + sex + age + high.educ.bl + household.income.bl + married.bl + scrn_commondx* sex

asr_scr_totprob_t scrn_commondx + race.4level + hisp + sex + age + high.educ.bl + household.income.bl + married.bl + scrn_commondx* sex

asr_scr_internal_t $\sim$ scrn_commondx + race.4level + hisp + sex + age + high.educ.bl + household.income.bl + married.bl + scrn_commondx * sex

asr_scr_external_t $~$ scrn_commondx + race.4level + hisp + sex + age + high.educ.bl + household.income.bl + married.bl + scrn_commondx * sex

asr_scr_adhd_t $\sim$ scrn_commondx + race.4level + hisp + sex + age + high.educ.bl + household.income.bl + married.bl + scrn_commondx * sex

anthro_bmi_calc scrn_commondx + race.4level + hisp + sex + age + high.educ.bl + household.income.bl + married.bl + scrn_commondx * sex 